\title{
Grain-Boundary Structure Effects on Intergranular Stress Corrosion Cracking of Alloy X-750
}

\author{
Brent L. Adams \\ b_I_adams@byu.edu \\ Ted Olsen \\ N. Panayotou \\ Y. Pan
}

Follow this and additional works at: https://scholarsarchive.byu.edu/facpub

Part of the Mechanical Engineering Commons

Original Publication Citation

Acta mater., Vol. 44, No. 12, pp. 4685-4695, 1996

\section{BYU ScholarsArchive Citation}

Adams, Brent L.; Olsen, Ted; Panayotou, N.; and Pan, Y., "Grain-Boundary Structure Effects on Intergranular Stress Corrosion Cracking of Alloy X-750" (1996). Faculty Publications. 680.

https://scholarsarchive.byu.edu/facpub/680

This Peer-Reviewed Article is brought to you for free and open access by BYU ScholarsArchive. It has been accepted for inclusion in Faculty Publications by an authorized administrator of BYU ScholarsArchive. For more information, please contact ellen_amatangelo@byu.edu. 


\title{
GRAIN-BOUNDARY STRUCTURE EFFECTS ON INTERGRANULAR STRESS CORROSION CRACKING OF ALLOY X-750
}

\author{
Y. PAN ${ }^{1}$, B. L. ADAMS 1 , T. OLSON ${ }^{2}$ and N. PANAYOTOU ${ }^{3}$ \\ 'Department of Materials Science and Enginecring, Carnegic Mellon University, Pittsburgh, PA \\ 15213-3890, ${ }^{2}$ Department of Mathematics, Michigan Technological University, Houghton, MI 49931 and \\ ${ }^{3}$ Lockheed Martin, Schenectady, NY 12301-1072, U.S.A.
}

http://www.sciencedirect.com/science/journal/13596454

\begin{abstract}
It is well known that the properties and behavior of grain boundaries are strongly affected by local chemistry and atomic structure. This paper focuses on the mesoscale description of grain boundary structure (i.e. the five crystallographic degrees of freedom) and correlations with intergranular stress corrosion cracking observed in Alloy X-750. Orientation imaging microscopy, coupled with serial polishing, is used to reveal mesoscale structure and the connectivity of the grain boundary network. The propensity for cracking is correlated with the coinçident site lattice (CSL) classification of grain boundary geometry, coupled with information about the orientation of the grain boundary plane. The data is interpreted to show that low-CSL boundaries (defined by the more restrictive Palumbo-Aust criterion), low-angle boundaries, and general boundaries with plane normals well off the principal stress axis, have low vulnerability to cracking.
\end{abstract}

\section{INTRODUCTION}

Intergranular stress corrosion cracking (ISCC) refers to cracking that propagates along the grain boundaries (GBs) of polycrystalline materials under the combination of applied stress and a corrosive environment. That the vulnerability of individual GBs to ISCC would be affected by local grain-boundary structure has long been suspected, but only recently have observations of local structure been systematically correlated with damage. These observations focus mainly on lattice misorientation as a convenient classification of the structure of GBs.

Here we shall use the term "lattice misorientation" when we refer to the smallest-angle axis-angle pair, selected from the large set of crystallographically equivalent axis-angle pairs, relating the adjacent lattices comprising the GB. Since the set of all physically distinctive GB types (i.e. the fundamental zone) is reckoned by five macroscopic degrees of freedom (three fixing lattice misorientation, and two fixing the inclination of the GB), it is clear that a focus on lattice misorientation alone constitutes a three-dimensional projection (hypersurface) through the much larger fundamental zone of distinctive GB types.

Within the context of this projection, recent studies have concluded that general high-angle GBs are much more vulnerable to ISCC damage than low-angle and special high-angle boundaries in some nickel-based alloys. The intergranular corrosion susceptibility of GBs in high-purity (polycrystalline) nickel was studied by Palumbo and Aust [1] in a corrosive environment consisting of $2 \mathrm{NH}_{2} \mathrm{SO}_{4}$ at $303^{\circ} \mathrm{K}$. Their central observation is that low-angle GBs, and vicinal boundaries with lattice misorientations near low- $\Sigma$ coincidence site lattices (CSLs) are found to be more resistant to localized corrosion than other high-angle general boundaries. On the basis of their data, these authors proposed a criterion for these resistant boundaries which has the form

$$
\delta \phi \leqslant 15^{\circ} \Sigma^{-5 / 6}
$$

where $\delta \phi$ denotes the allowable angle of deviation from the exact CSL misorientation. This is to be compared with the widely used (and less-restrictive) criterion of Brandon [2], which has the form

$$
\delta \phi \leqslant 15^{\circ} \Sigma^{-1 / 2} .
$$

Crawford and Was [3] assessed the vulnerability of GBs in Alloy 600 to ISCC damage in argon and high-purity water at $633^{\circ} \mathrm{K}$. Using a classification similar to that of Palumbo and Aust [1], they separated observed GBs into three groups: (i) low-angle boundaries, (ii) CSL boundaries, and (iii) general (random) high-angle boundaries. Using selected area channeling patterns to orient the lattice, they found that low-angle boundaries are rarely damaged, and that CSL boundaries are only half as susceptible to ISCC compared to general high-angle boundaries.

Although the classification set selected in these pioneering studies is rather coarse, and does not 
explicitly incorporate information about boundary plane orientation and connectivity, the results nevertheless suggest a tendency for small-angle and low- $\Sigma$ boundaries to be resistant to ISCC damage under certain environmental conditions. It follows that the distribution and connectivity of GB types could potentially affect the overall ISCC performance of these materials. Since it is also known that the distribution of GB types can be substantially altered by processing variations [4], it is useful to consider the engineered alteration of GB structure as a potential vehicle for improving ISCC performance in nickel-based alloys.

Here we report on a study of Alloy X-750. The aim was to investigate the role of $\mathrm{GB}$ structure in ISCC damage, with particular attention to crack propagation and the relationship of GB normal orientation (relative to the direction of maximum tensile stress), in addition to GB geometry. This was accomplished by refining the classification set to explicitly include the boundary normal orientation as a variable. The new method of orientation imaging microscopy (OIM) [5] has been used to rapidly determine the crystallographic character of GBs; this has been coupled with precision serial sectioning to reveal the full five parameters of structure for each exposed boundary. An important advantage is that many GBs can be examined in a realistic time frame, and that the full (mesoscopic) structure can be accessed from the experiments.

\section{EXPERIMENTAL METHODS}

The sample studied is a standard compact tension test specimen. ISCC was developed on the basis of a pre-existing notch which was subsequently fatigue precracked before exposure to the corrosive environment. The precrack, of length $1.9 \mathrm{~mm}$, was developed under low stress (stress intensity less than $1 / 3$ of the level initially induced during the corrosion experiment) to prevent fatigue damage or initial compressive stress at the crack tip. The corrosion test was performed under a constant displacement condition, using a bolt to induce opening of the notch (wedge opening loading). The initial stress intensity factor was $49.4 \mathrm{MPa} \mathrm{m}^{-1 / 2}$, corresponding to a tensile stress of $30 \%$ of yield. The stress intensity decreases as the crack propagates through the material.

The test environment consisted of high-temperature $\left(633^{\circ} \mathrm{K}\right)$ de-aerated water. The $\mathrm{pH}$ of the solution was 10 . No electrochemical potential was applied to the sample.

The test period spanned 336 days, and a final crack length of $9.93 \mathrm{~mm}$ on one side and $9.0 \mathrm{~mm}$ on the other was observed. This corresponds to approximately 100 average grain diameters. The average crack growth rate falls in the range 2.69$3.42 \times 10^{-10} \mathrm{~m} / \mathrm{s}$. Of the total crack length, the first half was found to exhibit a mixture of intergranular and transgranular fracture modes. The second half, however, was found to exhibit a completely intergranular character. This may suggest a transition of mode due to the decreasing stress intensity with increasing crack length.

OIM was used to reveal the microstructure adjacent to the crack path. Figure 1 shows the OIM record from one particular section, nominally perpendicular to the crack. In the left representation of the record, diffraction image quality has been used to highlight the grain structure, the crack path and regions of high plastic deformation being highlighted by dark grey scale. In the right representation of the record, the OIM record has been used to highlight the extensive occurrence of GBs exhibiting $\Sigma 3$ misorientation character. A direct comparison of optical metallography and OIM is shown in Fig. 2. Clearly the optical metallography fails to contrast many of the annealing twins present in the structure.

In order to reveal the three-dimensional geometry of the GB network, which is obscured by the opacity of the material under optical or scanning electronoptical examination, a serial sectioning procedure was adopted. The approach is straightforward; the sample is polished down perpendicular to the observation surface by a measurable amount. Vickers microhardness indents were introduced into the surface at strategic locations. Upon polishing, the reduced exposed area of these indents is measured. From the known angles of the indenter it is then possible to estimate the thickness of the removed layer. Figures 3 and 4 show a pair of optical micrographs with indentations before and after this polishing procedure. The remnants of the original indentations (and the new indentations introduced to facilitate the next removal procedure) are evident. The aim was to remove $10 \mu \mathrm{m}$ with each successive procedure. In total, ten layers of material were exposed according to the plan shown in Fig. 5. Each of the three strata were fully characterized using OIM; approximately 90,000 single orientation measurements were obtained from each layer as the basis for the OIMs. The step size between measurement points was selected to be $10 \mu \mathrm{m}$; scanning occurred over hexagonal grids. The ten sections were separated into three groups to insure that different grains were exposed in three separate strata. At least four microhardness indentations were employed on each section to facilitate the estimates of removal thickness. Based upon an analysis of possible errors, the limiting resolution of the GB inclination angle is believed to be $\sim 9^{\circ}$. Although it would be highly desirable to improve the resolution of the inclination angle, this was not possible with the available experimental facilities.

\section{MATERIALS CHARACTERIZATION}

The sample analysed is of Alloy X-750. The major material contents are listed in Table 1 . The chromium content is $15.5 \%$, which is about the same level found 
in Alloy $600(16 \%)$. The sample was rolled at high temperature, solution-annealed at $1366^{\circ} \mathrm{K}$ for one hour, and then age-hardened at $977^{\circ} \mathrm{K}$ for $20 \mathrm{~h}$. It is a matter of practical experience that this heat treatment gives the best resistance to ISCC in both pressurized water reactor and boiling water reactor environments [6].

From standard optical metallography the
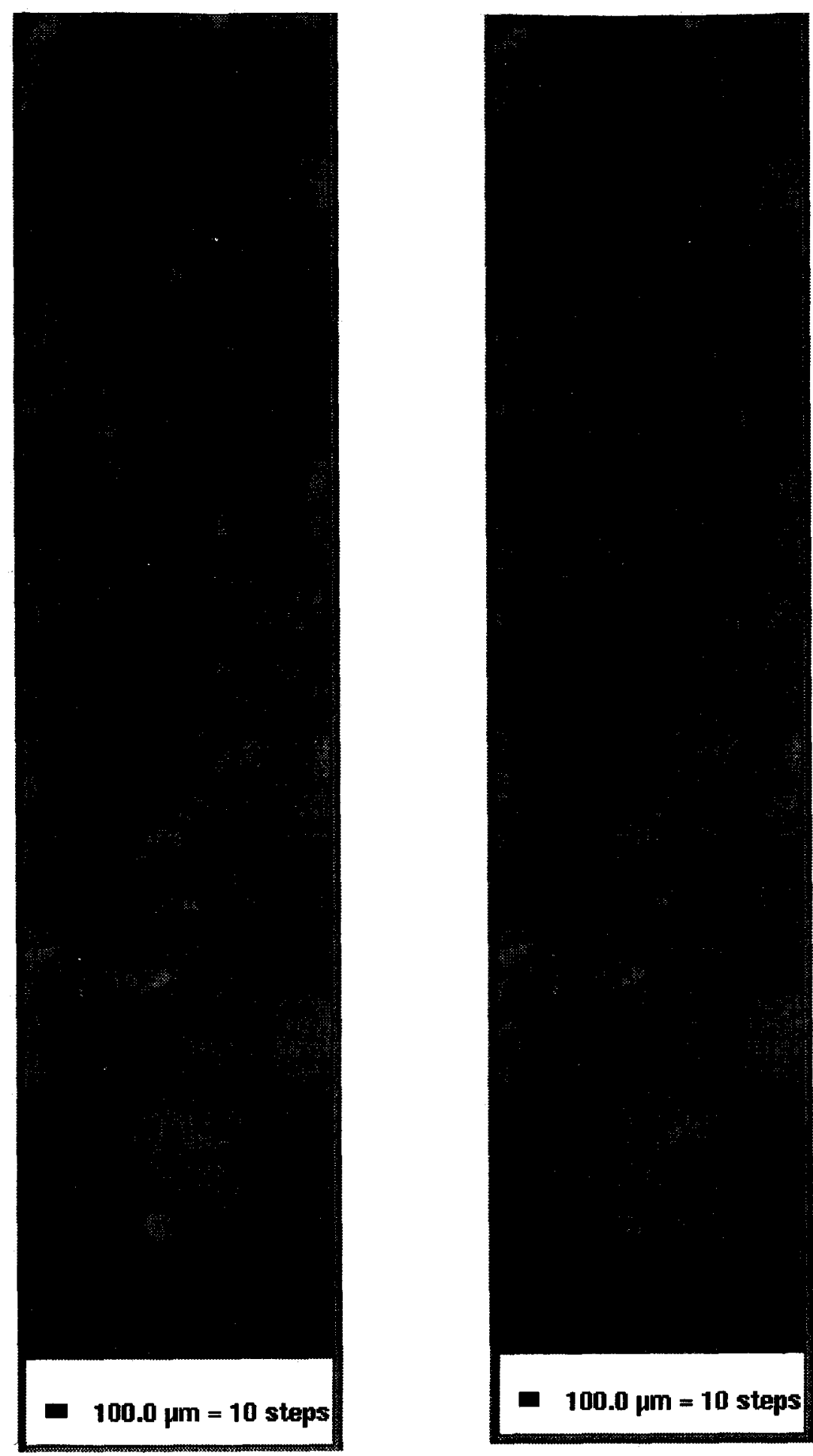

Fig. 1. Orientation imaging micrographs of Alloy X-750 showing microstructure near ISCC damage. The left image is formed directly from the measured index of imaging quality, and clearly shows the crack line. In the right image black lines denote $\Sigma 3$ boundaries according to Brandon's criterion. 

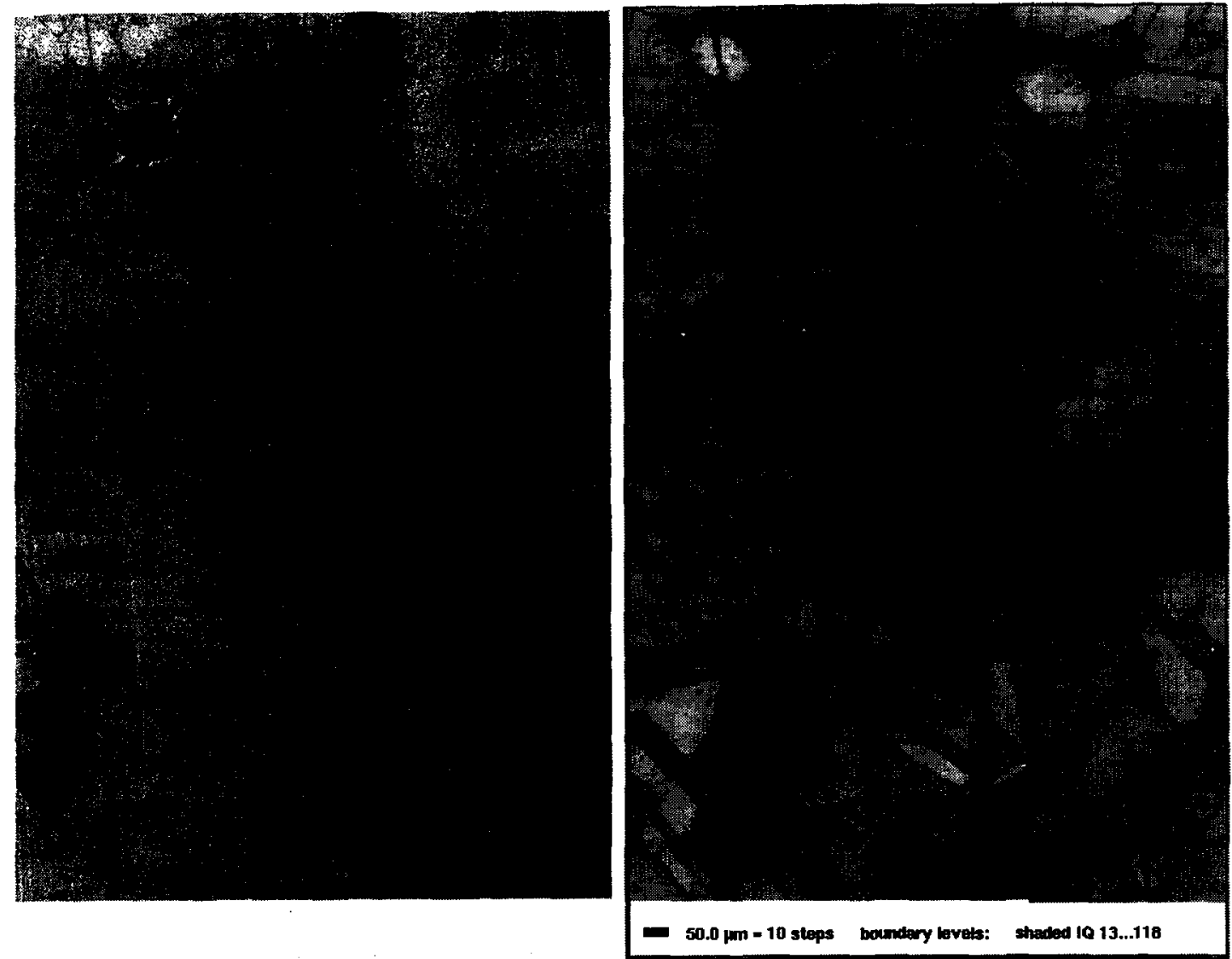

Fig. 2. Direct comparison of optical (left) and OIM (right, obtained from $\sim 30,000$ single orientation measurements) imaging of an intergranular stress corrosion crack in Alloy X-750.

measured average grain size of the material is $114 \mu \mathrm{m}$, but the more sensitive OIM revealed the average grain size to be $89 \mu \mathrm{m}$. The material exhibits a Knoop hardness of 350 using a 200 gram force. Measured yield stresses are $744 \mathrm{MPa}$ at $294^{\circ} \mathrm{K}$ and $689 \mathrm{MPa}$ at $633^{\circ} \mathrm{K}$, which is the temperature at which corrosion testing was performed. Young's modulus for this material is $207 \mathrm{GPa}$ at $294^{\circ} \mathrm{K}$, and Poisson's ratio is 0.29 .

Alloy X-750 is a precipitation hardened material; the main hardening agent is the intermetallic $\gamma^{\prime}$ phase $\left[\mathrm{Ni}_{3}(\mathrm{Al}, \mathrm{Ti})\right]$. In addition, a prominent feature of the microstructure is the carbide precipitation $\left(\mathrm{M}_{7} \mathrm{C}_{3}\right.$ and $\mathrm{M}_{23} \mathrm{C}_{6}$ ) on (primarily) the GBs. In the sample of interest, $60-70 \%$ of the GBs are observed to contain carbide precipitates. Although the mechanism is still a matter of debate [7-9], it is generally held that the presence of carbide decoration improves the local resistance to ISCC.
The orientation and misorientation textures of the sample were both obtained using OIM [10]. Plotting programs made use of the standard series expansion method, with Gaussian smoothing [11]; the series was truncated at order 22 in the generalized spherical harmonics. Sections through the space of Euler angles are shown for the orientation distribution function (ODF) in Fig. 6. The maximum intensity that occurred is 1.93 times random; this suggests a very weak, nearly random distribution of grain orientations. (The Euler angles used are those defined by Bunge [11].)

The misorientation distribution function (MODF) is shown in Fig. 7. It is well known that the MODF is substantially impacted by the formation of twins during annealing. A maximum intensity of 7.8 times random is found centered upon the $\Sigma-3$ misorientation. This peak is very broad, with more than 20 degrecs of spread. It can be secn from cquations (1) 
and (2) that many of the misorientations in this broad peak do not satisfy either the Brandon or the Palumbo-Aust criteria for specialness. A secondary peak can be identified at the location of the $\Sigma-9$ misorientation. (The fundamental zone of the cubic misorientation space in the space of Euler angles, and its relationship to the coincidence site lattice model, has been described by Zhao and Adams [12].)

It is useful to examine the misorientation distribution of the material in the broader context of CSL theory. Using the spread criteria of PalumboAust [equation (1)] and Brandon [equation (2)], the total set of 31.612 measured GBs have been classified into small-angle boundaries $(\Sigma 1), \Sigma 3$ boundaries (presumably twins), other low- $\Sigma$ special boundaries ( $\Sigma 5 ., \ldots, \Sigma 49$ ), and all other random high-angle boundaries (HABs). The results are shown in Table 2 . Other details about the distribution of CSL boundaries in the sample are shown in Fig. 8. Clearly, the percentage of boundaries that can be classified as "special". meaning vicinal boundaries in the $\Sigma 1-\Sigma 49$ range, depends upon the criterion chosen. Choosing Brandon's criterion suggests that $57 \%$ of the boundaries are special, whereas the more restrictive Palumbo-Aust criterion lowers this figure to $49 \%$. The percentage of boundaries near the $\Sigma 3$ misorientation is found to be about $35 \%$ by either criterion.
Obviously, both criteria give the same fraction of small-angle boundaries.

Twinning plays a dominant role in the formation of the observed microstructure. The low stacking fault energy of Alloy X-750 $\left(\sim 0.12 \mathrm{~J} / \mathrm{m}^{\prime}\right)$ enables the extensive formation of annealing twins. Several models have been proposed for their formation [13-16] although none explain all of the observed features. It can easily be demonstrated by numerical simulation that twinning leads to a certain degree of randomization of the orientation distribution. This may in fact account for the weak orientation distribution observed in our samples. Perhaps less well appreciated is the observation that the intersections of twins with general high-angle boundaries has an appreciable probability of altering the character of the new boundary in the direction of low-angle or CSL boundaries. In the present work we have simulated the possible effects of the multiple variants of twinning upon some 554 (initially) random high-angle boundaries lying upon the observed crack path. The result is that approximately $16 \%$ of these particular boundaries are altered to become special boundaries in the $\Sigma 1-\Sigma 49$ range, on the basis of Brandon's criterion. Of course we would expect that twinning could change low-angle boundaries into high-angle random boundaries, but this

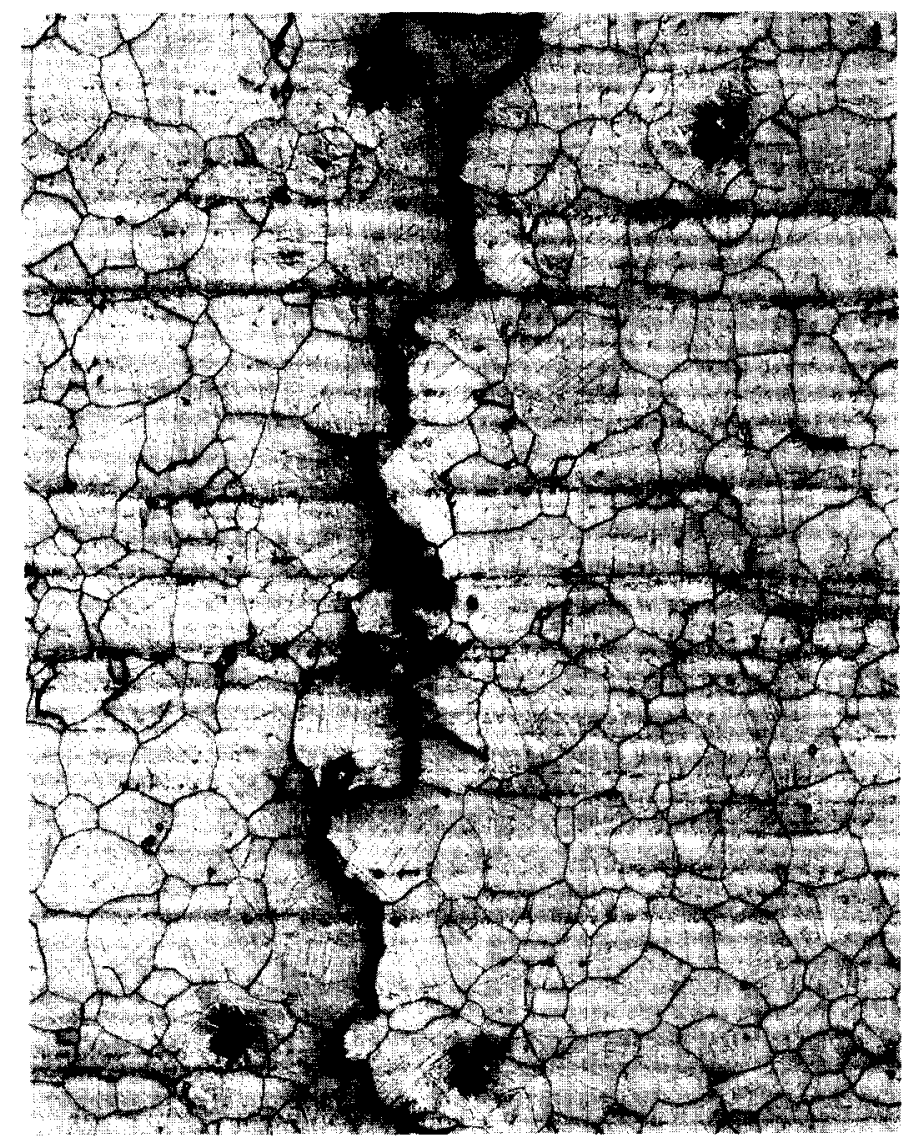

Fig. 3. Micrograph showing initial indents. The diagonals of the indents are about $80 \mu \mathrm{m}$ long. 


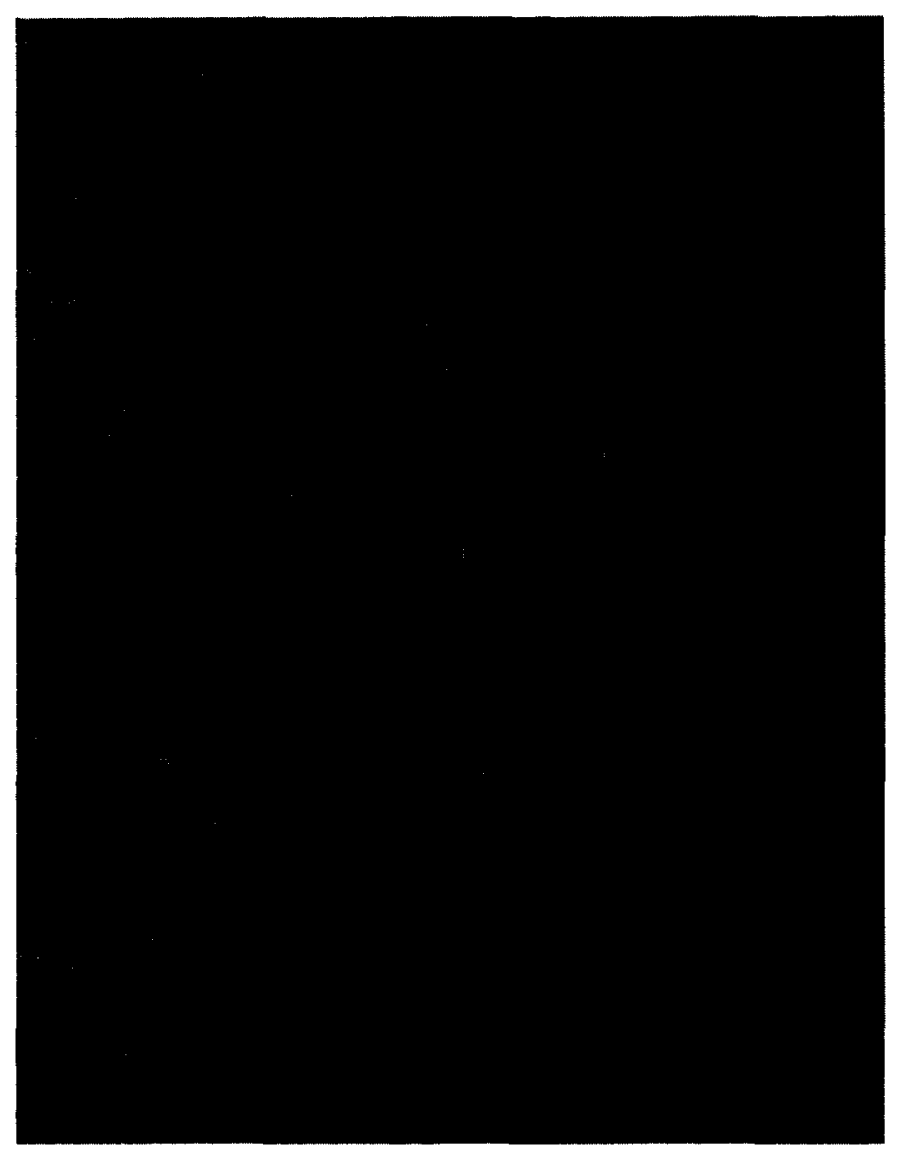

Fig. 4. Micrograph showing remains of initial indents and newly put indents, with diagonal distance of new indents about $80 \mu \mathrm{m}$.

phenomenon was not observed in our measurements of the microstructure. Successive twinning (of twins) will lead to the formation of other $\Sigma 3^{n}$ boundaries [4].

\section{DESCRIPTION OF THE SELECTIVITY OF CRACK PROPAGATION IN THE MICROSTRUCTURE}

\subsection{Sensitivity to lattice misorientation}

Altogether, 818 triple junctions in the measurement set were exposed to the advancing crack. It is useful to consider the decisions the advancing crack tip makes as it propagates on either one leg or the other of each triple junction. Of the 818 triple junctions encountered, 541 junctions included a GB with $\Sigma 3$ character. In no case was a $\Sigma 3$ boundary found to crack. Thus, whenever one branch of a triple junction exhibited 23 misorientation the crack followed the other branch or was arrested. (Several instances were observed where the crack was arrested at a triple junction, re-emerging one grain further along the forward direction of propagation. These arrests were always associated with triple junctions containing one $\Sigma 3$ boundary, and another boundary unfavorably oriented for crack propagation.) Of the remaining 277 triple junctions, only two low-angle $(\Sigma 1)$ boundaries were found to be cracked. Upon closer examination of these two boundaries it was found that one had misorientation of $14.5^{\circ}$ and the other $14.6^{\circ}$. Thus, both fell near the limit of what is normally considered to be a small angle. Figure 9 shows the distribution of misorientation angles, for the entire set of triple junctions, separated into cracked and uncracked populations. For comparison, the theoretical random distribution of cubic misorientations $[17,18]$ has been superimposed. Clearly, both

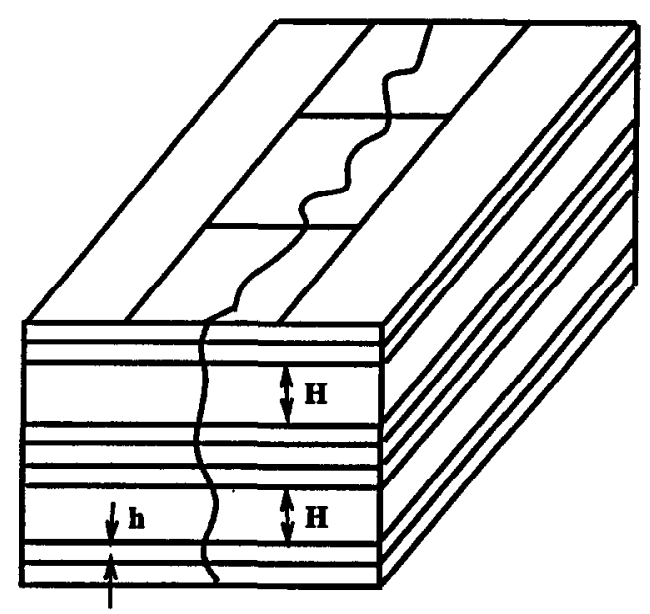

Fig. 5. The (parallel) serial sectioning scheme: $H \approx 500 \mu \mathrm{m}$, $h \approx 10 \mu \mathrm{m}$. 
Table 1. Compositions of Alloy X-750 alloys

\begin{tabular}{lcccccccccc}
\hline Alloy & UNS No. & $\mathrm{C} \dagger$ & $\mathrm{Cr}$ & $\mathrm{Cu}$ & $\mathrm{Fe}$ & $\mathrm{Mo}$ & $\mathrm{Nb}$ & $\mathrm{Ni}$ & $\mathrm{Ti}$ & Others \\
\hline $\mathrm{X}-750$ & $\mathrm{~N} 07750$ & 0.08 & 15.5 & - & 7.0 & - & 0.9 & $70 \ddagger$ & 2.5 & $\mathrm{Al}: 00.7$ \\
\hline
\end{tabular}

†Maximum, †minimum

populations follow roughly the random distribution, but strong (somewhat counter-posed) oscillations are present.

Among the total set of observed triple junctions encountered by the advancing crack, 42 cracked boundaries fell in the low- $\Sigma$ category $(\Sigma 1-\Sigma 49)$ according to Brandon's criterion. The distribution of these boundaries is shown in Fig. 10. It is interesting that when the more restrictive Palumbo-Aust criterion is applied, only three of these boundaries are conserved in the low- $\Sigma$ class. A complete listing of these 42 boundaries, including several details about each boundary, is given in Table 3. The three boundaries still satisfying the requirements for low $\Sigma$ CSL classification under the Palumbo-Aust criterion are listed as boundaries 11,33 and 42 .

\subsection{Sensitivity to crystal plane orientation}

Although the possible errors in resolving boundary normal orientation are estimated to be quite large $\left(\sim 9^{\circ}\right)$, we have nevertheless considered crystal plane orientations at cracked and uncracked boundaries. Figure 11 shows the measured crystal plane orientations in the standard triangle for the $\Sigma$ 3-disoriented boundaries found in one representative OIM. Although substantial clustering is observed near the (111) coherent plane, associated with symmetrical twin boundaries, the scatter in the unit triangle is quite remarkable. Some secondary clustering can be imagined for other locations (e.g. along the (100)-(111) or (110)-(111) ribs of the standard triangle), but it is evident that the experimental limitations do not facilitate any strong conclusions. The fact that not even a single $\Sigma 3$ boundary was found to be cracked suggests that
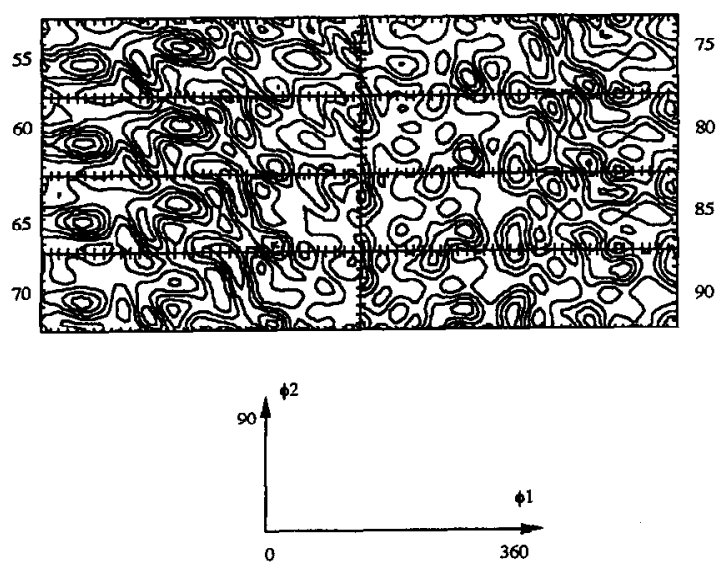

Fig. 6. ODF shown in Euler space. Contour level lines are $0.2,0.4,0.6, \ldots, 2.0$. Maximum value $=1.9$. crystal plane orientation may not play a significant role for this class of boundaries.

Figure 12 focuses on the populations of boundaries found in the set of 818 triple junctions along the crack path. The distribution of measured crystal plane orientations for cracked and uncracked boundaries is shown in the standard triangle. Within the limitations of these experiments, no clustering is found in either population.

It is instructive to consider the set of 42 cracked boundaries that fall in the low- $\Sigma$ CSL classification. With reference to Table 3, columns 6 and 7 list the measured $(h k l)$ planes associated with (adjacent) grains 1 and 2 which form the GB-these are compared with the densely packed planes (column 5) predicted from CSL theory; $\eta_{1}$ and $\eta_{2}$ denote the (minimum) angle between the measured crystal normal and the densely packed normal predicted by the theory; $\eta_{\mathrm{c}}$ denotes the maximuin allowable deviation of misorientation allowed by Brandon's criterion [equation (2)]; columns 8 and 9 in Table 3 present the ratios $\eta_{1} / \eta_{2} / \eta_{\mathrm{c}}$. Only those boundaries with ratios of the order one or less should be considered to be vicinal boundaries in the low $-\Sigma$ classification
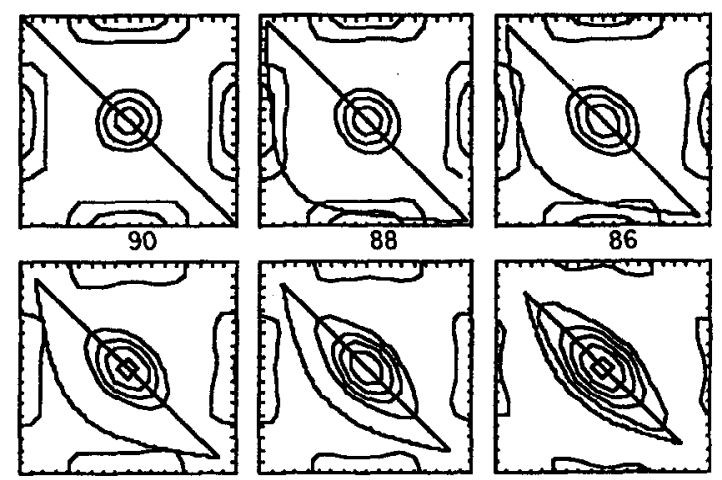

84
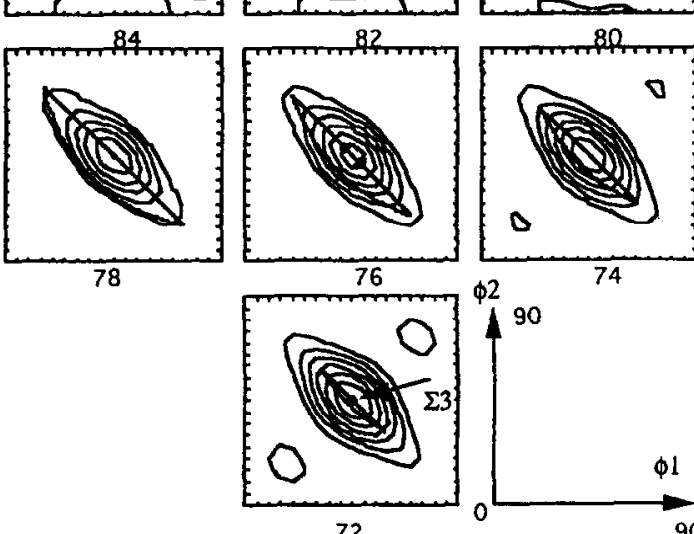

72

Fig. 7. MODF shown in Euler space. Contour level lines are $1,2,3,4,5,6,7$. Maximum value $=7.8$. 
Table 2. Distribution of CSL boundary percentages in bulk material

\begin{tabular}{lccccc}
\hline & \multicolumn{2}{c}{ Brandon } & & \multicolumn{2}{c}{ Palumbo-Aust } \\
\cline { 2 - 3 } GB type & Number of segments & Percentage & & Number of segments & Percentage \\
\hline$\Sigma 1$ & 2555 & $11.3 \%$ & & 2555 & $11.3 \%$ \\
$\Sigma 3$ & 8056 & $35.7 \%$ & & 7874 & $34.9 \%$ \\
$\Sigma 5+\ldots+\Sigma 49$ & 2244 & $9.9 \%$ & & 638 & $2.8 \%$ \\
Random HAB & 9731 & $43.1 \%$ & & 11,519 & $51.0 \%$ \\
Total & 22,586 & $100 \%$ & & 22,586 & $100 \%$ \\
\hline
\end{tabular}

when the GB plane is also given consideration. It is evident from Table 3 that none of the measured boundaries have ratios less than one. On this basis all of the 42 cracked boundaries found in the low- $\Sigma$ CSL classification by misorientation alone could be rejected from the same classification when their GB normal orientations are considered.

\subsection{Sensitivity to crack propagation direction}

We have also considered the sensitivity of cracking to the orientation which the boundary plane makes relative to the macroscopic direction of crack propagation. The results for the set of 818 triple junctions on the crack are shown in Fig. 13. Plotted is the (minimum) angle which the direction of forward advance of the boundary plane makes with the macroscopic (average) direction of forward crack advance. This is an angle that lies in the range $[0, \pi]$. Those boundaries which lie closest to the forward propagation direction are, as cxpected, most vulncrable to damage. Although clearly some cracking occurs in a sense opposite to the forward cracking direction, no example was found making an angle greater than $120^{\circ}$, and most of the cracked boundarics exhibited angles less than $60^{\circ}$.

4.4. Classification of grain boundaries according to their susceptibility for intergranular stress corrosion cracking

The experimental results suggest a comparison of different classification schemes which would order

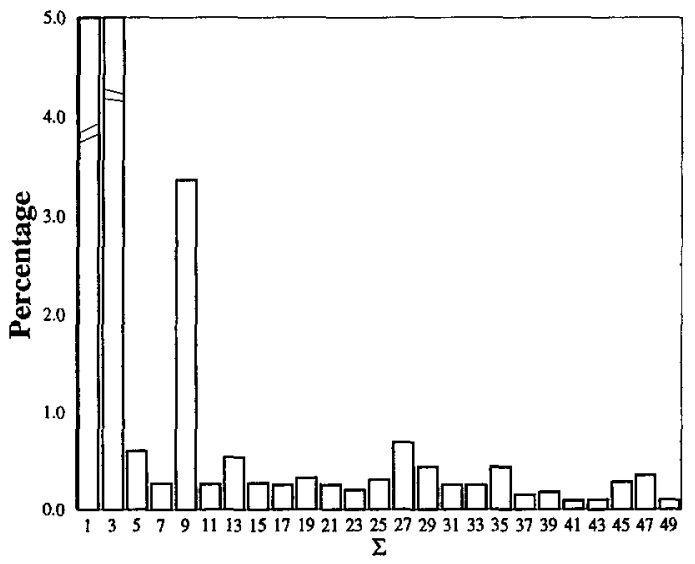

Fig. 8. CSL boundary distributions in bulk material. The percentages of $\Sigma 1$ and $\Sigma 3$ boundaries are $11.3 \%$ and $35.7 \%$, respectively.
GBs according to their relative resistance to ISCC. Here we compare three approaches to classification with the record of ISCC damage in the microstructure.

The simplest classification is suggested by the classical Read-Shockley relationship between lattice misorientation, $\theta$, and GB energy [19]:

$$
\gamma_{\mathrm{gb}}=\gamma_{\mathrm{o}} \theta(A-\ln \theta) \text {. }
$$

In this relationship $\gamma_{0}$ and $A$ depend upon material and geometrical constants. We assume that the change in energy of the system, $\Delta U$, owing to the formation of a crack of area $\Delta A$, is given by the ideal energy of fracture

$$
\Delta U=\left(\gamma_{1}+\gamma_{2}-\gamma_{\mathrm{gb}}\right) \Delta A
$$

where $\gamma_{1}$ and $\gamma_{2}$ are the specific surface energies associated with the new free surfaces created when the GB separates. Since surface energies are generally larger than GB energies, higher values of $\Delta U$ would generally be associated with lower GB energies, and lower susceptibility to cracking. Thus, an ordering of susceptibility for cracking can be computed, based upon the Read-Shockley relationship for each of the triple junctions observed along the crack. Branching decisions based upon the Read-Shockley relationship were compared with the record of decisions among the 277 measured triple junctions which did not involve the $\Sigma 3$ boundary type. It was found that the Read-Shockley relationship predicted the correct boundary for crack advancement in only 140 of the measured cases, or approximately $50 \%$ of the time.

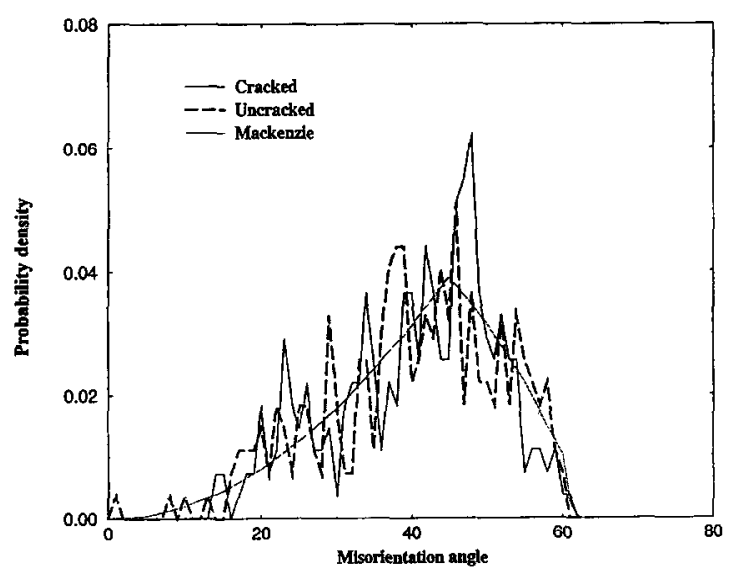

Fig. 9. Minimum misorientation angle distributions of cracked and uncracked boundaries. 


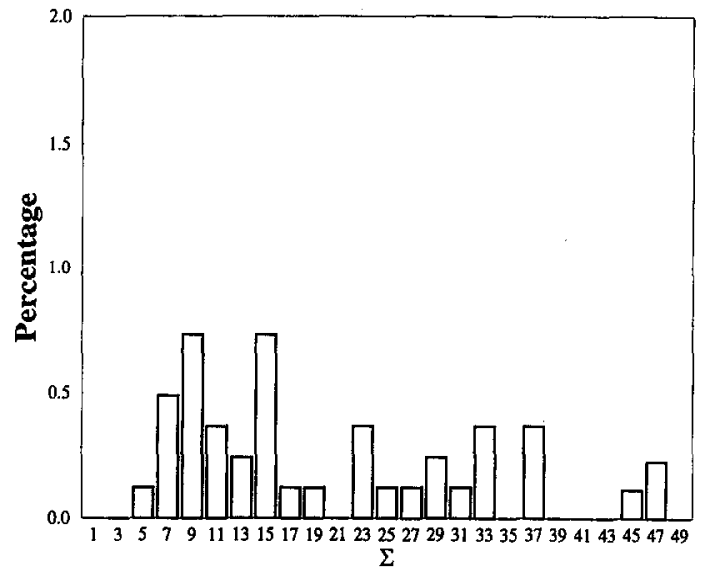

Fig. 10. Cracked CSL boundary distributions in the material. The percentages were calculated from a total of 818 boundaries.

It might be supposed that the Read-Shockley classification would be much less reliable for high-angle boundaries, given its motivation based upon the dislocation densities associated with low-angle boundaries. For this reason an augmented classification has been considered which incorporates

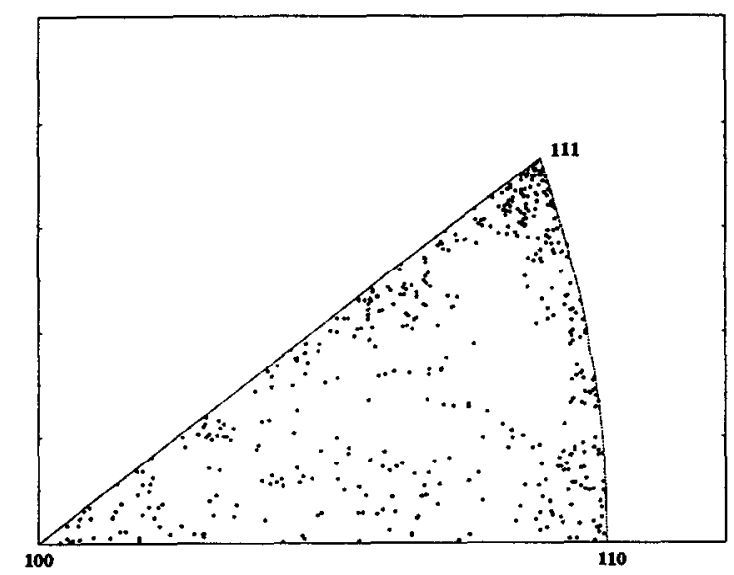

Fig. 11. Crystalline plane distributions in standard triangles for $\Sigma 3$ boundaries.

a reduction of the surface energy for all interfaces that fall in the low- $\Sigma$ classification $(\Sigma 5-\Sigma 29)$, relative to other high-angle boundaries. This was implemented by considering the 33 triple junctions from Table 3 which fell in this low- $\Sigma$ range. Each boundary in this category was given a surface energy lower than the other boundaries with misorientations greater

Table 3. Characteristics of cracked CSL boundaries

\begin{tabular}{|c|c|c|c|c|c|c|c|c|c|c|c|c|}
\hline \multirow{2}{*}{$\frac{\text { No. }}{1}$} & \multirow{2}{*}{$\frac{\Sigma(B)}{7}$} & \multirow{2}{*}{$\frac{\theta}{33.7}$} & \multirow{2}{*}{$\frac{\Delta \theta / \omega_{\Sigma}(P-A)}{0.99}$} & \multirow{2}{*}{$\frac{\text { Twin plane }}{321}$} & \multicolumn{3}{|c|}{$h_{1} k_{1} l_{1}$} & \multicolumn{3}{|c|}{$h_{2} k_{2} l_{2}$} & \multirow{2}{*}{$\frac{\eta_{1} / \eta_{\mathrm{c}}}{1.8}$} & \multirow{2}{*}{$\frac{\eta_{2} / \eta_{\mathrm{c}}}{2.4}$} \\
\hline & & & & & 0.09 & 0.76 & 0.64 & 0.05 & -0.78 & 0.63 & & \\
\hline 2 & 9 & 39.4 & 0.96 & 221,421 & 0.50 & 0.81 & -0.30 & -0.54 & 0.76 & 0.36 & $2.6,1.4$ & $1.8,2.4$ \\
\hline 3 & $31 a$ & 18.0 & 0.6 & 651 & 0.15 & 0.88 & 0.46 & 0.54 & -0.76 & 0.36 & 4.6 & 5.4 \\
\hline 4 & $29 \mathrm{~b}$ & 44.5 & 0.73 & 432 & 0.35 & 0.65 & -0.67 & 0.34 & 0.91 & 0.22 & 3.0 & 6.7 \\
\hline 5 & 9 & 38.2 & 0.93 & 221,421 & -0.88 & 0.36 & 0.32 & 0.78 & 0.03 & 0.62 & $1.5,4.3$ & $4.5,3.4$ \\
\hline 6 & 11 & 53.7 & 0.84 & 311,332 & 0.54 & 0.84 & 0.07 & -0.84 & 0.52 & 0.16 & $5.3,4.3$ & $4.5,3.4$ \\
\hline 7 & $25 b$ & 51.8 & 0.75 & 543 & -0.74 & -0.14 & 0.66 & 0.85 & 0.07 & 0.53 & 5.7 & 7.2 \\
\hline 8 & 15 & 48.3 & 0.95 & 521 & 0.84 & 0.53 & 0.11 & 0.70 & 0.27 & 0.66 & 2.9 & 5.6 \\
\hline 9 & 23 & 42.2 & 0.96 & 631 & 0.09 & 0.95 & 0.29 & -0.95 & 0.05 & 0.30 & 3.5 & 3.6 \\
\hline 10 & $37 b$ & 44.1 & 0.71 & 831 & 0.99 & 0.14 & 0.04 & 0.87 & 0.01 & 0.49 & 4.9 & 4.2 \\
\hline 11 & $17 \mathrm{~b}$ & 61.1 & $0.30(0.77)$ & 322,433 & -0.34 & 0.79 & 0.51 & 0.94 & 0.21 & 0.27 & $2.5,2.5$ & $7.4,6.5$ \\
\hline 12 & 11 & 48.9 & 0.40 & 311,332 & 0.77 & 0.39 & 0.51 & 0.61 & 0.38 & -0.70 & $3.2,2.2$ & $4.7,1.2$ \\
\hline 13 & $21 \mathrm{a}$ & 22.9 & 0.93 & 541 & 0.08 & 0.74 & 0.67 & 0.08 & 0.76 & 0.65 & 1.4 & 1.4 \\
\hline 14 & $19 \mathrm{a}$ & 24.5 & 0.64 & 331,611 & 0.82 & 0.08 & 0.57 & 0.94 & 0.14 & 0.29 & $3.7,7.3$ & $6.9,1.8$ \\
\hline 15 & 15 & 49.2 & 0.78 & 521 & 0.10 & 0.83 & 0.55 & -0.50 & 0.86 & 0.06 & 3.3 & 2.8 \\
\hline 16 & 15 & 48.8 & 0.67 & 521 & 0.33 & -0.69 & 0.64 & 0.26 & 0.87 & 0.41 & 5.6 & 1.3 \\
\hline 17 & 23 & 40.0 & 0.52 & 631 & 0.02 & 0.86 & 0.51 & 0.44 & -0.89 & 0.09 & 2.6 & 1.0 \\
\hline 18 & $47 a$ & 37.2 & 0.69 & 763 & 0.65 & 0.30 & 0.70 & -0.88 & 0.48 & 0.08 & 2.5 & 8.1 \\
\hline 19 & 15 & 48.3 & 0.88 & 521 & 0.31 & 0.54 & 0.78 & 0.64 & 0.75 & 0.19 & 3.8 & 4.7 \\
\hline 20 & 7 & 40.7 & 0.59 & 321 & -0.89 & -0.41 & 0.18 & 0.07 & 0.98 & 0.21 & 1.8 & 4.3 \\
\hline 21 & 7 & 38.6 & 0.76 & 321 & 0.68 & 0.69 & 025 & 0.73 & 0.58 & 0.36 & 1.7 & 1.3 \\
\hline 22 & 23 & 39.4 & 0.81 & 631 & 0.02 & 0.83 & 0.55 & -0.78 & 0.34 & 0.53 & 3.2 & 4.2 \\
\hline 23 & $37 \mathrm{~b}$ & 43.7 & 0.41 & 831 & 0.47 & 0.85 & 0.23 & 0.20 & 0.97 & 0.11 & 4.5 & 3.6 \\
\hline 24 & 5 & 34.9 & 0.69 & 210,310 & 0.98 & -0.11 & 0.14 & 0.85 & 0.38 & 0.36 & $1.8,2.9$ & $3.2,3.4$ \\
\hline 25 & $13 b$ & 31.5 & 0.94 & 431 & 0.63 & 0.33 & 0.70 & 0.52 & 0.56 & 0.65 & 2.9 & 4.4 \\
\hline 26 & 11 & 53.9 & 0.93 & 311,332 & -0.81 & 0.57 & 0.14 & 0.93 & 0.34 & 0.11 & $4.1,4.3$ & $2.5,5.6$ \\
\hline 27 & 7 & 36.2 & 0.78 & 321 & 0.98 & 0.08 & 0.17 & 0.38 & 0.88 & 0.28 & 4.5 & 1.8 \\
\hline 28 & $33 b$ & 33.7 & 0.76 & 741 & 0.35 & 0.92 & 0.16 & -0.36 & 0.78 & 0.51 & 3.4 & 5.6 \\
\hline 29 & $33 b$ & 35.5 & 0.82 & 741 & -0.32 & 0.90 & 0.18 & 0.06 & -0.95 & 0.30 & 3.6 & 4.9 \\
\hline 30 & $45 c$ & 52.2 & 0.43 & 754 & 0.38 & -0.86 & 0.32 & 0.70 & 0.68 & 0.21 & 5.5 & 6.7 \\
\hline 31 & 9 & 50.3 & 0.6 & 221,421 & 0.65 & 0.71 & -0.28 & 0.74 & 0.46 & 0.47 & $1.4,3.2$ & $3.3,3.6$ \\
\hline 32 & $13 a$ & 20.6 & 0.94 & 320,510 & 0.97 & -0.19 & -0.12 & 0.57 & 0.79 & 0.20 & $5.6,1.6$ & $2.8,6.5$ \\
\hline 33 & 15 & 47.7 & $0.31(0.76)$ & 521 & 0.75 & 0.66 & 0.02 & 0.38 & 0.91 & 0.16 & 5.5 & 1.1 \\
\hline 34 & $35 b$ & 44.0 & 0.48 & 653 & 0.01 & 0.74 & 0.67 & 0.89 & 0.45 & 0.02 & 8.5 & 9.2 \\
\hline 35 & $21 a$ & 37.1 & 0.97 & 541 & 0.73 & -0.44 & 0.52 & 0.94 & 0.33 & 0.10 & 5.3 & 5.9 \\
\hline 36 & 9 & 38.9 & 0.97 & 221,421 & 0.79 & 0.47 & 0.38 & 0.27 & 0.95 & 0.17 & $7.8,2.1$ & $5.9,2.2$ \\
\hline 37 & $29 \mathrm{~b}$ & 48.2 & 0.84 & 432 & -0.13 & 0.93 & 0.36 & 0.28 & 0.94 & 0.20 & 7.4 & 7.8 \\
\hline 38 & $21 \mathrm{~b}$ & 46.6 & 0.99 & 421 & 0.39 & 0.90 & 0.19 & 0.36 & 0.29 & 0.89 & 1.2 & 1.9 \\
\hline 39 & 15 & 49.0 & 0.91 & 521 & 0.61 & 0.78 & -0.11 & 0.72 & 0.57 & -0.37 & 4.3 & 5.1 \\
\hline 40 & $43 c$ & 60.8 & 0.74 & 533,655 & 0.28 & 0.92 & -0.26 & 0.86 & 0.46 & 0.21 & $7.8,8.4$ & $6.7,9.1$ \\
\hline 41 & $13 b$ & 29.1 & 0.86 & 431 & 0.28 & -0.96 & 0.02 & 0.75 & -0.61 & 0.25 & 5.5 & 1.2 \\
\hline 42 & 9 & 40.2 & $0.41(0.84)$ & 221,421 & 0.85 & -0.41 & 0.33 & 0.95 & 0.31 & 0.10 & $3.7,1.4$ & $5.9,2.1$ \\
\hline
\end{tabular}


than $15^{\circ}$. It was found that this modified ReadShockley relationship then predicted 148 of the 277 measured branching decisions correctly, or about $53 \%$. Evidently, since the number of low $-\Sigma$ boundaries is small, we would not expect to see a large improvement in the percentage of correct branching predictions. (It must also be remembered that measurements of the GB plane normal orientation suggest that many, if not all of the boundaries listed in Table 3 , are not true CSL boundaries.)

It is quite evident that any classification which would be successful in predicting branching probabilities must consider the orientation of the GB with respect to the nominal macroscopic crack plane and direction of crack propagation. Here we propose an augmented classification which takes into account a broader range of the salient features observed in the measured data set. We consider that all small-angle boundaries with misorientations less than $14^{\circ}$, and all $\Sigma 3$ misorientations (regardless of the crystallographic
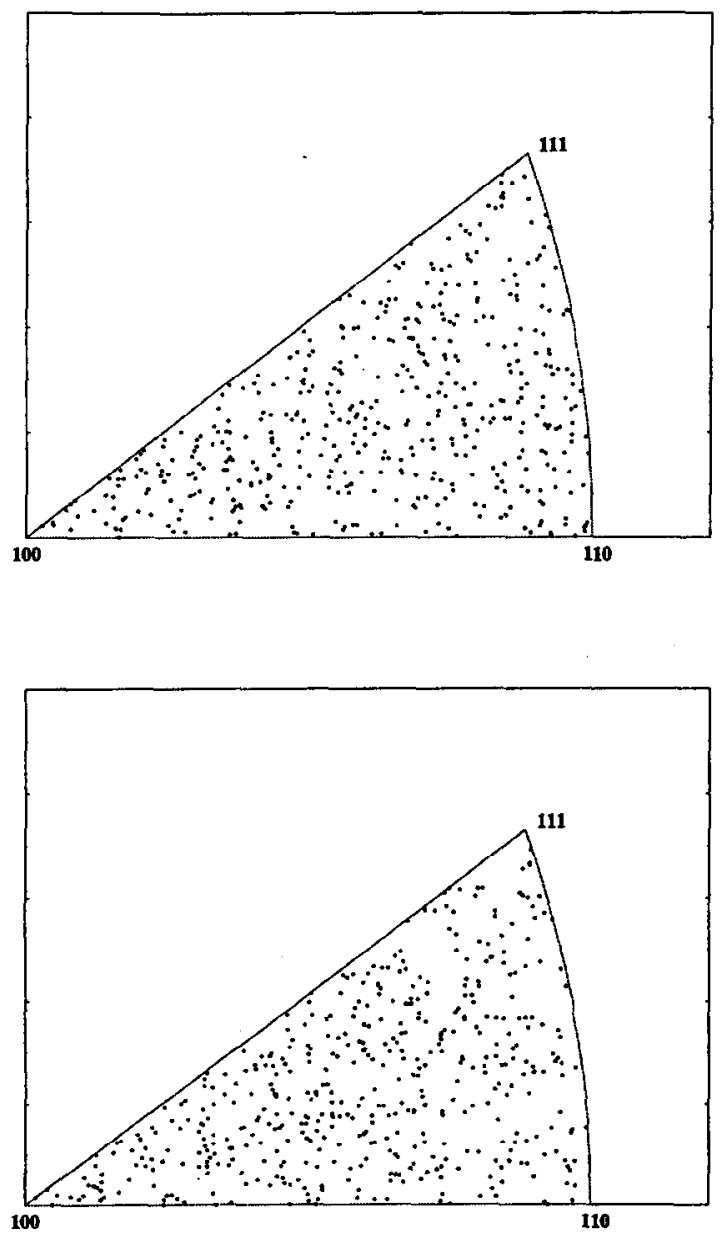

Fig. 12. Crystalline plane distributions in standard triangles for cracked boundaries (top) and uncracked boundaries (bottom).

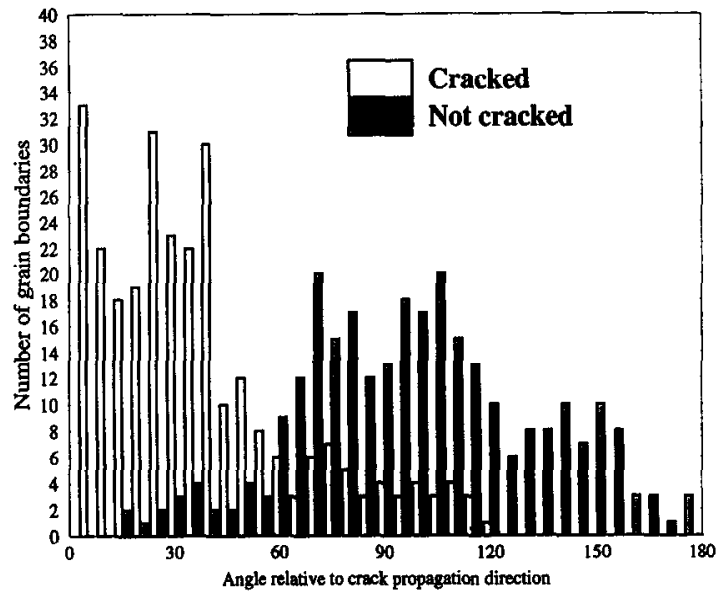

Fig. 13. Grain boundary normal distribution with respect to crack propagation direction (polar angle $\Pi$ as the horizontal axis).

character of the boundary plane) have the lowest probability of cracking. Denote this class of boundaries as class $\mathrm{A}$. The experimental evidence suggests that other low- $\Sigma$ boundaries in the range $\Sigma 5-\Sigma 29$ may have lower probabilities for cracking than general high-angle boundaries, although insufficient experimental evidence exists to make a strong case. This second class of boundaries is labeled $\mathbf{B}$. The third category is the category of other high-angle GBs, and is labeled C. Furthermore, we have subdivided each category into three ranges of boundary plane angle: $i \in\left(0,30^{\circ}\right), j \in\left(31,60^{\circ}\right), m \in$ $\left(61,90^{\circ}\right), n \in\left(91,180^{\circ}\right)$. The classification suggested by the experimental record can be expressed as

$$
\begin{array}{r}
P_{\mathrm{A} n}<P_{\mathrm{A} m}<P_{\mathrm{A} j}<P_{\mathrm{A} i}<P_{\mathrm{B} n}<P_{\mathrm{B} m}<P_{\mathrm{B} j}<P_{\mathrm{B} i} \\
<P_{\mathrm{C} n}<P_{\mathrm{C} m}<P_{\mathrm{C} j}<P_{\mathrm{C} i}
\end{array}
$$

where, for example $P_{\mathrm{B} m}$ denotes the probability of cracking a boundary found within the low $-\Sigma$ category (type B), whose boundary plane falls in the range $\left(61,90^{\circ}\right)$ (subcategory $m$ ). Using this new classification scheme, 237 of the 277 triple junction decisions not involving the $\Sigma 3$ boundary type were correctly predicted, or about $86 \%$. If the entire set of 818 triple junctions is considered, this classification gives a $95 \%$ success rate. Failures of this classification set are usually associated with triple junctions where both branches fall in the same element of the classification set.

It is evident that considering the geometrical orientation of GBs substantially improves the prediction of branching decisions at triple junctions. The classification set introduced in equation (5) is still very coarse, and it is likely that refinements are possible. However, within the context of the limited data available, we concluded that further attention to refinement of the set would be of questionable value. 


\section{SUMMARY AND DISCUSSION}

We have examined the microstructure of a sample of Alloy X-750 damaged by ISCC, after fatigue precracking, in a high-temperature environment of deaerated water. The main focus of the study has been to study potential correlations between the geometrical structure of GBs and their propensity for cracking. Using OIM, coupled with calibrated serial polishing, all five geometrical parameters of GB structure were estimated. Four of these angular parameters can be measured to an uncertainty of about $1^{\circ}$, but the angle of inclination of the boundary could only be estimated to an accuracy of about $9^{\circ}$.

Regarding the ex-situ record of ISCC propagation in the material, we find that the general high-angle boundaries are most susceptible to cracking, given that the direction of forward crack propagation of the macroscopic crack lies within about $120^{\circ}$ of the local crack planc. Small-anglc (absolute minimum misorientation $<14^{\circ}$ ) and $\Sigma 3$ GBs were not observed to crack, regardless of the orientation of the local plane with respect to the forward crack direction. This observation is all the more significant in the light of the observation that a broad distribution of plane normals is observed for $\Sigma 3$ GBs. Some CSL boundaries lying in the range $\Sigma 5-\Sigma 49$, purely on the basis of lattice misorientation, were found to crack; however, when the crystallographic character of the crack plane, and/or the more restrictive misorientation criterion of Palumbo and Aust are considered, the evidence suggests that very few (if any) true CSL boundaries in this range actually cracked. These observations are in harmony with the earlier studies of Palumbo and Aust [1] and Crawford and Was [3] on other nickel-based materials which did not explicitly consider the orientation of the GB plane.

On the basis of the data collected in these observations, an ordering of the susceptibilities to ISCC damage was proposed, [embodied in inequality (5)]. This ordering classification takes into account both the lattice misorientation and the inclination of the $G B$ relative to the nominal forward cracking direction. All boundaries have been classified into one of 12 categories. On this basis the branching decisions at $95 \%$ of the observed triple junctions along the crack path are correctly predicted. It is evident that a finer classification could probably be devised which would have an even higher success ratio; however, with the current experimental limitations in measuring GB inclination angles, and the limited number of triple junctions observed along the advancing crack (818 in the present study) it is not likely that a great improvement could be achieved.

Acknowledgements-This work was supported by the Department of Energy under Grant No. DE-FG02-88ER 45355. TO was supported by the National Science Foundation during her 1993-94 stay at the Institute for Advanced Studies (Princeton) under grant DMS-9304580. The authors wish to acknowledge helpful discussion with Carol Nichols, John Hack, Gino Palumbo, Karl Aust, and Gary Was.

\section{REFERENCES}

1. G. Palumbo and K. T. Aust, Acta metall. mater. 38, 2343 (1990).

2. D. G. Brandon, Acta metall. 14, 1479 (1966).

3. D. C. Crawford and G. S. Was, Metall. Trans. 23A, 1195 (1992)

4. V. Y. Gertsman, K. Tangri and R. Z. Valiev, Acta metall. mater, 42, 1785 (1994).

5. B. L. Adams, S. I. Wright and K. Kunze, Metall. Trans. 24A, 819 (1993).

6. N. Sridhar and G. Cragnolino, in Stress-Corrosion Cracking, (edited by R. H. Jones). ASM International (1992).

7. D. Vaughn, Acta metall. 18, 183 (1970)

8. K. Yamanaka, in Proc. 6th Int. Symp. Environmental Degradation of Materials in Nuclear Power SystemsWater Reactors, (edited by R. E. Gold and E. P. Simonen), p. 105. The Metallurgical Society of AIME Pittsburgh (1993).

9. S. M. Bruemmer, L. A. Charlot and C. H. Henager, in Corrosion '87, paper no. 88. NACE (1987).

10. B. L. Adams, Mater. Sci. Engng A166, 59 (1993).

11. H.-J. Bunge, Texture Analysis in Materials Science: Mathematical Methods, p. 180. Butterworths, London (1982).

12. J. Zhao and B. L. Adams, Acta Crystallogr. A44, 326 (1988)

13. S. Dash and N. Brown, Acta metall. 11, 1067 (1963).

14. R. E. Fullman and J. C. Fisher, J. appl. Phys. 22, 1350 (1951).

15. H. Gleiter, Acta metall. 17, 1421 (1969).

16. M. A. Meyers and L. E. Murr, Acta metall. 26, 951 (1978).

17. J. K. MacKenzie, Biometrika 45, 229 (1958).

18. J. K. MacKenzie, Acta metall. 12, 223 (1964).

19. W. Shockley and T. W. Read, Jr, Phys. Rev. 78, 275 (1950). 